\title{
Validation of a Saliva-Based Test for the Molecular Diagnosis of SARS-CoV-2 Infection
}

\author{
Michela Bulfoni (D, , ${ }^{1,2}$ Emanuela Sozio, ${ }^{3}$ Barbara Marcon, ${ }^{4}$ Maria De Martino, ${ }^{1}$ \\ Daniela Cesselli, ${ }^{1,2}$ Chiara De Carlo, ${ }^{3}$ Romina Martinella, ${ }^{4}$ Angelica Migotti, ${ }^{4}$ \\ Eleonora Vania, ${ }^{3}$ Agnese Zanus-Fortes, ${ }^{3}$ Jessica De Piero, ${ }^{3}$ Emanuele Nencioni, ${ }^{5}$ \\ Carlo Tascini, ${ }^{1,3}$ Miriam Isola, ${ }^{1}$ and Francesco Curcio $\mathbb{D}^{1,4}$ \\ ${ }^{1}$ Department of Medicine, University of Udine, Udine, Italy \\ ${ }^{2}$ Institute of Pathology, ASU FC, Udine, Italy \\ ${ }^{3}$ Infectious Disease Unit, Department of Medicine, ASU FC, Udine, Italy \\ ${ }^{4}$ Department of Laboratory Medicine, ASU FC, Udine, Italy \\ ${ }^{5}$ Biofarma Srl, Mereto di Tomba Udine, Italy
}

Correspondence should be addressed to Michela Bulfoni; michela.bulfoni@uniud.it

Received 2 September 2021; Revised 22 November 2021; Accepted 13 December 2021; Published 7 January 2022

Academic Editor: Stamatios E. Theocharis

Copyright (c) 2022 Michela Bulfoni et al. This is an open access article distributed under the Creative Commons Attribution License, which permits unrestricted use, distribution, and reproduction in any medium, provided the original work is properly cited.

Background. Since the beginning of the pandemic, clinicians and researchers have been searching for alternative tests to improve the screening and diagnosis of the SARS-CoV-2 infection. Currently, the gold standard for virus identification is the nasopharyngeal (NP) swab. Saliva samples, however, offer clear, practical, and logistical advantages but due to a lack of collection, transport, and storage solutions, high-throughput saliva-based laboratory tests are difficult to scale up as a screening or diagnostic tool. With this study, we aimed to validate an intralaboratory molecular detection method for SARS-CoV-2 on saliva samples collected in a new storage saline solution, comparing the results to NP swabs to determine the difference in sensitivity between the two tests. Methods. In this study, 156 patients (cases) and 1005 asymptomatic subjects (controls) were enrolled and tested simultaneously for the detection of the SARS-CoV-2 viral genome by RT-PCR on both NP swab and saliva samples. Saliva samples were collected in a preservative and inhibiting saline solution (Biofarma Srl). Internal method validation was performed to standardize the entire workflow for saliva samples. Results. The identification of SARS-CoV-2 conducted on saliva samples showed a clinical sensitivity of $95.1 \%$ and specificity of $97.8 \%$ compared to NP swabs. The positive predictive value (PPV) was $81 \%$ while the negative predictive value (NPV) was $99.5 \%$. Test concordance was $97.6 \%$ (Cohen's Kappa $=0.86 ; 95 \%$ CI $0.81-0.91$ ). The LoD of the test was 5 viral copies for both samples. Conclusions. RT-PCR assays conducted on a stored saliva sample achieved similar performance to those on NP swabs, and this may provide a very effective tool for population screening and diagnosis. Collection of saliva in a stabilizing solution makes the test more convenient and widely available; furthermore, the denaturing properties of the solution reduce the infective risks belonging to sample manipulation.

\section{Introduction}

The coronavirus disease 19 (COVID-19) pandemic driven by the novel severe acute respiratory syndrome coronavirus 2 (SARS-CoV-2) is an aggressive and potentially deadly dis- ease firstly appeared in Wuhan, China, in 2019 and then rapidly spread worldwide $[1,2]$.

SARS-CoV-2 is transmitted by exposure to infectious respiratory fluids [2-6]. The early detection of infected subjects plays a crucial role in their isolation to stop the spread 
of the infection $[3,6]$. Adequate sample collection and transportation are important elements for the laboratory diagnosis of SARS-CoV-2 [7]. A specimen that is not collected properly may be an explanation for false negative results, as in the case of nasopharyngeal swab sampling $[5,8]$.

The reverse transcription polymerase chain reaction (RTPCR) in nasopharyngeal (NP) swab samples is the current gold standard in detection of SARS-CoV-2 infection $[5,9,10]$.

Specimen collection currently required specialized staff, exposes personal to a high risk of infection, and causes discomfort to patients $[11,12]$. Furthermore, there is a need for a simpler and less invasive methods to make it a mass test [13-17]. An additional limiting factor is the inadequate sampling that may result in both lower specimen quantity and in lower test sensitivity $[5,18]$.

To overcome these limitation, other types of biological matrices are currently under investigation [7, 18]. Among these, an easily and simple-to-use alternative specimen for the detection of SARS-CoV-2 in the diagnosis of COVID-19 is saliva samples $[5,15,19]$. Saliva represents a wide and homogenous resource for genomic information, useful for studying the disease status [19-22].

COVID-19 diagnostic on saliva samples overcomes the need for a trained technician and also reduces the potential risk of infection $[13,20,21,23]$. Benefits of the saliva testing include the noninvasiveness, the practical storage, and the easier sample collection in particular fragile patient populations (e.g., paediatric, noncollaborative, and elderly patients) [22-24]. Overall, saliva testing can reduce infection spread and costs and can provide an easier access for patients $[6,25]$.

Considering all benefits, researchers and clinicians concur that the diagnosis and prevention of COVID-19 disease using human saliva is to be better explored [5]. The noninvasive nature and ease of collection have made saliva the fluid of choice not only for diagnostic but also for more important health surveillance purposes [13, 20, 26]. Saliva is suggested to cause spread via droplet infection, appearing to be more transmissible $[17,17,27]$. For example, novel investigations on saliva samples were recently proposed to understand SARS-CoV-2 variant spread [19, 22, 24, 28].

Evidences on the use of saliva for the detection of SARSCoV-2 are exponentially increasing, representing saliva a very attractive, readily available, and repeatable biological matrix [20, 28]. RT-PCR assays using saliva swab have been approved in the United States and in Japan [7, 11]. In the US, the FDA has already approved saliva-based collection methods, especially in emergencies [4].

Several papers have been published on the possible use of saliva as an alternative diagnostic tool to identify SARSCOV-2 infection [19, 24, 28]. The comparison of saliva and NP molecular tests has shown discrepancies between studies $[15,18,29]$. In some studies, nasopharyngeal swab samples showed a greater sensitivity; others found contrary results, while in some cases saliva has proved equally effective as a diagnostic specimen $[16,18,26,29,30]$.

Prior works have taken into account the need of stabilization buffers for collecting saliva samples, considering that the enzymatic composition could represent a negative factor for nucleic acid stabilization and protease inhibition [31].
Saliva is stickiness and difficult to stabilize due to its muco-proteins and enzymatic composition [31, 32]. In particular, its endogenous nucleases primarily contribute to the RNA degradation and lead to varying degrees of interference during the analytical steps, such as nucleic acid extraction and amplification by PCR [20, 29]. Furthermore, saliva viscosity may reduce sample's recovery affecting downstream applications [20,31]. Saliva collection techniques and timing, conditioning, and delays in processing raw saliva samples represent the major causes for failure to apply saliva as a validated diagnostic source [20, 31, 33, 34].

Moreover, despite the hot topic, no systematic studies are available yet, in particular concerning saliva collection and analysis validation based on both patients and asymptomatic or healthy groups.

The aim of this study was to analyse the feasibility of saliva as an alternative diagnostic specimen for detecting SARS-CoV-2 infection both in patients with confirmed diagnosis of COVID-19 and in healthy people who had to undergo screening tests. The objectives were (i) to establish and validate a standardized and reproducible saliva collection method, using a saline-based solution, in order to produce high-quality RNA to improve the performance of downstream molecular investigations; (ii) to compare results of the RT PCR-based molecular test on saliva samples versus NP swabs, in terms of concordance, clinical sensitivity, and specificity; and (iii) to investigate potential association between test results and COVID-19.

\section{Materials and Methods}

2.1. Study Design and Participants. In this observational study, a total of 1161 participants (age $\geq 18$ ) were enrolled between October 2020 and May 2021. Among these, 156 were patients with confirmed diagnosis of COVID-19 (cases), admitted to the Infectious Disease Clinic of Udine, and 1005 were asymptomatic people who had to undergo screening tests (controls). Both groups were tested for the detection of SARSCoV-2 with NP swab and in saliva specimens.

The aim of the study was to compare results of the RT PCR-based molecular test in detecting SARS-CoV-2 RNA on saliva samples and on the recommended NP swab, which is considered the gold standard. For hospitalized patients, we also looked for any correlation between the positivity of the two tests (in terms of cycle threshold) and clinical severity, defined as a composite outcome of admission to Intensive Care Unit (ICU) or death.

All samples collected were anonymized using an alphanumeric identification code, and the study was approved by the Local Ethics Committee.

2.2. Nasopharyngeal and Saliva Sample Collection. NP specimens were collected by midturbinate swabbing of both nares and the posterior pharynx, avoiding the tongue. A flocked swab (ESwab Copan) was used for the collection of all NP clinical samples and handled as recommended in international guidelines $[1,4,7]$. For saliva collection, a noninvasive method was executed by making self-collected specimen in a tube container. The conserving solution contained 
in the saliva collection tubes was provided by Biofarma Srl, and it is protected by intellectual property. The specific saline composition and the $\mathrm{pH}$ value made this solution optimal for the stabilization and conservation of viral RNA in saliva. Furthermore, the high saline concentration takes a denaturing effect on protein and as consequence on cell structure and proliferation. For these reasons, when saliva comes into contact with the solution, proteins and cells are disrupted hindering the viral activity. Hence, saliva samples can be considered safer for the healthcare personnel.

The subjects enrolled simply spat into the container with the conserving solution and were not asked to perform oral hygiene, to collect the sample at particular times of the day or between meals.

2.3. Viral RNA Extraction. Viral RNA was extracted and purified starting from NP swabs and saliva samples using the Virus/Pathogen Kit (Qiagen), on an automated Qiasymphony extractor, according to the manufacturer's instruction. The purification procedure is designed to ensure safe, reproducible handling of potentially infectious samples and comprises three steps: lyse, bind, and wash, taking the advantage of a magnetic bead principle.

RNA extracted was finally eluted into a multiwall plate in a volume of $60 \mu \mathrm{L}$ of Buffer AE (Qiagen).

2.4. One Step Real Time PCR Assay. A reverse transcriptase, one-step real time PCR was employed for the detection of SARS-CoV-2 Envelope viral protein (coded by the E-gene). Primer sequences were selected according CDC's guidelines and purchased from Roche (Roche). The amplification was performed using LightCycler ${ }^{\circledR}$ Multiplex RNA Virus Master (Roche), following the company's instructions. For each reaction, $5 \mu \mathrm{L}$ of template was loaded. For each reaction, a synthetic RNA positive control and a no template control were used. RT-PCR and analyses were performed employing the LightCycler 480 (Roche) instrument. For each amplification reaction, $\mathrm{Cp}$ values were computed. We considered test results as positive when cycle threshold $(\mathrm{Ct})$ values were $<36$, according to the assay limit of detection (LoD) defined.

2.5. Assay Validation. The evaluation of assay accuracy was performed by testing 7 different dilutions of a patient positive sample $(\mathrm{Cp}=29)$, with 10 replicates each. Serial dilutions (from $1.00+E^{6}$ to 2 copies) of a synthetic RNA control were also computed in order to define the expected concentration values. The precision of our analytical procedure was established as repeatability and intermediate precision and examined using the one-way ANOVA, according to UNI ISO 5725-2: 2020 guidelines.

The linear range of RT-PCR was established with a series of 7-step dilutions, tested in 10 replicates each.

Assay LoD was determined by utilizing different dilution of samples with known Cp values. The performance of saline collecting solution was assessed on 25 samples of saliva, matched with the fresh saliva samples collected from the same patients. The stability of saliva samples collected in the saline solution was tested at 24 and 48 hours, at two dif- ferent storage temperatures (room temperature RT, $18-25^{\circ} \mathrm{C}$ and $\left.4^{\circ} \mathrm{C}\right)$.

2.6. Statistics. Sensitivity, specificity, positive, and negative predictive values and their 95\% confidence intervals (CI) were calculated to assess diagnostic performance. Kappa coefficient was presented to estimate agreement between swab and saliva RT-PCR test results, with its 95\% CI.

Bland-Altman analysis was used to compare the $\mathrm{Ct}$ values between swab and saliva results.

Descriptive statistics for categorical variables are presented as number (percent) and for continuous variables as mean \pm standard deviation (SD) or median (interquartile range (IQR)). Normality was assessed using Shapiro-Wilk test. Comparisons between categorical variables were performed using the Chi-square or Exact Fisher test, as appropriate. For continuous variables, comparisons between two groups were done using the $t$-test or Mann-Whitney $U$ test; comparisons among groups were done using ANOVA or Kruskal-Wallis test, as appropriate. For multiple comparisons, Bonferroni's correction was applied.

For hospitalized patients, Cox regression was used to estimate association between death or admission to ICU and swab Ct; after the assumptions were verified; the same analysis was performed for saliva Ct. Performance of the models was evaluated by Harrell's concordance index (C-index). All analyses were performed by the STATA 16 statistical software, and statistical significance was set at $p<0.05$.

\section{Results}

3.1. Population and Diagnostic Performances of the Tests. In this study, a total of 1161 participants were enrolled, who provided both NP swabs and saliva specimens for the molecular detection of SARS-CoV-2.

Among these, 156 (13.4\%) were patients admitted to the Infectious Disease Clinic with confirmed diagnosis of COVID-19 (cases), while 1005 (86.6\%) were asymptomatic people who had to undergo screening tests (controls).

The overall prevalence for SARS-CoV-2 molecular detection determined on NP swab was $8.9 \%$ (103/1161, $95 \%$ IC 7.3-10.7) while on saliva specimen was $10.4 \%$ (121/ 1161, 95\% IC 8.7-12.3).

Among the 156 patients certainly affected by COVID-19 (cases), an average of 12 days (IQR 9-16 days) has passed between the onset of symptoms and the test performed for the purpose of comparison saliva to NP swab. 62.2\% of cases (97/156) showed a SARS-CoV-2 positive NP swab while $71.8 \%(112 / 156)$ resulted positive if tested on saliva specimen. In the group of people who had to undergo screening tests (controls), the positivity with the classical swabs and with saliva specimen was reached in $0.6 \%(6 / 1005)$ and in $0.9 \%$ (9/1005), respectively.

Using the NP swab as the reference gold standard, the clinical sensitivity and specificity reached by saliva was 95.1\% (95\% CI $89-98.4 \%)$ and $97.8 \%$ (95\% CI $96.8-$ $98.6 \%)$, respectively. The positive predictive value (PPV) 
was $81 \%$ (95\% CI 72.9-87.6\%) while the negative predictive value (NPV) was 99.5\% (95\% CI 98.9-99.8\%).

Analysis of concordance was conducted comparing results obtained from saliva samples and the NP goldstandard swab. The agreement between the two tests was 97.6\% with a Cohen's Kappa coefficient of 0.862 (95\% CI 0.812-0.912).

Concordance between tests was observed to be associated with subject's age: in the concordant group, the medium age was 50 years (IQR 30-59), while in the discordant was 70 years (IQR 61-76) with a significant difference between the two groups $(p<0.001)$. Table 1 shows demographic and clinical characteristics of patients with confirmed diagnosis of COVID-19 (cases).

In particular, the comparison between group with both saliva and swab concordant test (both positive or both negative) results to be significant ( $p=0.006$; Bonferroni's correction was applied).

Concordance of RT-PCR results was also associate with onset of symptoms before test (days) $(p=0.0036)$, as shown in Table 2.

Data regarding $\mathrm{Ct}$ values were available for 112/156 molecular tests based on saliva and for 71/156 NP swabs. Comparison of $\mathrm{Ct}$ values between saliva and swab in this group of patients showed no significant differences.

The matching among positive samples is shown in Figure 1, where Ct values have been paired by patients.

Taking the advantage of the Bland-Altman (B\&A) plot, the relationship between the $\mathrm{Ct}$ average value reached by the 2 tests ( $x$ axis), and their difference ( $y$ axis) was explored, in order to assess methods' concordance. Data presented were randomly distributed in the plot between the upper and lower limit of agreement (Figure 2).

As regards data on $\mathrm{Ct}$ values, where available in both salivary and NP swabs test ( $n=71$ cases), we evaluated if the values found in the two tests were associated with clinical severity (composite outcome considered as orotracheal intubation or death).

The RT-PCR Ct value of both salivary and NP swab tests results to be significantly associated with COVID-19 severity in both tests as shown in Table 3. In particular, we found that higher $\mathrm{Ct}$ values, meaning very low levels of viral RNA load found, were a protective factor.

Furthermore, the Harrell's $\mathrm{C}$ showed a better performance of the model in the case of the NP swab test $(0.738$ vs. 0.659).

3.2. Preanalytical Considerations: Stabilizing Properties of the Saline Solution. Saliva collected in the saline solution from 25 patients was used to preliminary evaluate its conserving properties. Comparing RT-PCR results obtained from both fresh and preserved saliva samples of a same patient, a gain of $28 \%$ positive tests $(N=7 / 25)$ was observed. Considering all paired results, a significant difference between $\mathrm{Ct}$ values emerged depending on the collection method (Figure 3). In particular, stabilized saliva samples showed significantly lower Ct $(p=0.006)$, with a median value of 27.9 (IQR 23.8-32.3) compared with results computed from fresh samples (median Ct 32, IQR 29-35).
TABle 1: Demographic and clinical features of the case group.

\begin{tabular}{lc}
\hline & Patients $(n=156)$ \\
\hline Age, median (IQR) & $72(62-77)$ \\
Days of symptoms before test, median (IQR) & $12(9-16)$ \\
Admission to ICU, $n(\%)$ & $34(21.8)$ \\
Death, $n$ (\%) & $29(18.6)$ \\
Admission to ICU or death, $n(\%)$ & $47(30.1)$ \\
\hline
\end{tabular}

TABle 2: Relationship between test agreement and onset of symptoms before test (days).

\begin{tabular}{lccc}
\hline $\begin{array}{l}\text { NP } \\
\text { swab }\end{array}$ & $\begin{array}{c}\text { Saliva } \\
\text { samples }\end{array}$ & $\begin{array}{c}N \text { of } \\
\text { observations }\end{array}$ & $\begin{array}{c}\text { Days of symptoms before test, } \\
\text { median (IQR) }\end{array}$ \\
\hline Negative & Negative & 38 & $14(12-17)^{*}$ \\
Negative & Positive & 20 & $15.5(11.5-18)$ \\
Positive & Negative & 5 & $13(13-13)$ \\
Positive & Positive & 89 & $11(8-14)^{*}$ \\
\hline
\end{tabular}

${ }^{*}$ Comparison between group with both positive saliva and swab concordant test (both positive or both negative) showed statistically significant difference in terms of days of symptoms before test $(p=0.006)$.

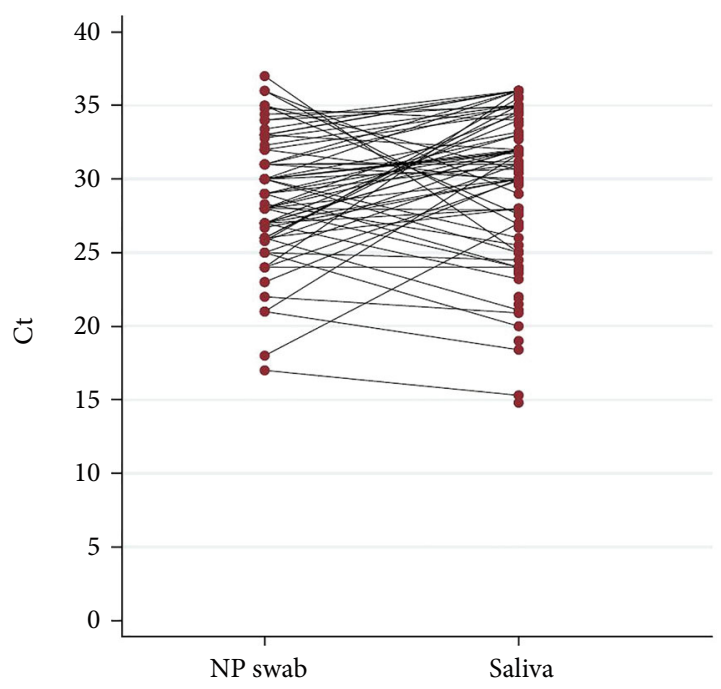

FIgURE 1: Ct values for paired samples in the 156 patients tested for SARS-CoV-2 infection.

Furthermore, the temporal stability of SARS-CoV-2 RNA collected in the preserving saline solution, measured at different holding temperatures $\left(20-25^{\circ} \mathrm{C}\right.$ and $\left.4^{\circ} \mathrm{C}\right)$, was about 48 hours. We found that SARS-CoV-2 viral load was reliably detected at similar levels regardless of the holding time and temperatures tested. We observed a $100 \%$ of sensitivity both at 24 and 48 hours, independently from storage temperature as confirmation that SARS-CoV-2 RNA was relatively stable. No positive sample lost was noticed. Our data indicates that viral RNA remains protected even at room temperature and at $4^{\circ} \mathrm{C}$ up to $48 \mathrm{~h}$ if collected in saline medium.

3.3. Validation of the Analytical Procedure. The accuracy of the RT-PCR method was measured by the coefficient of variation $(\mathrm{CV})$ between measurements obtained from 7 


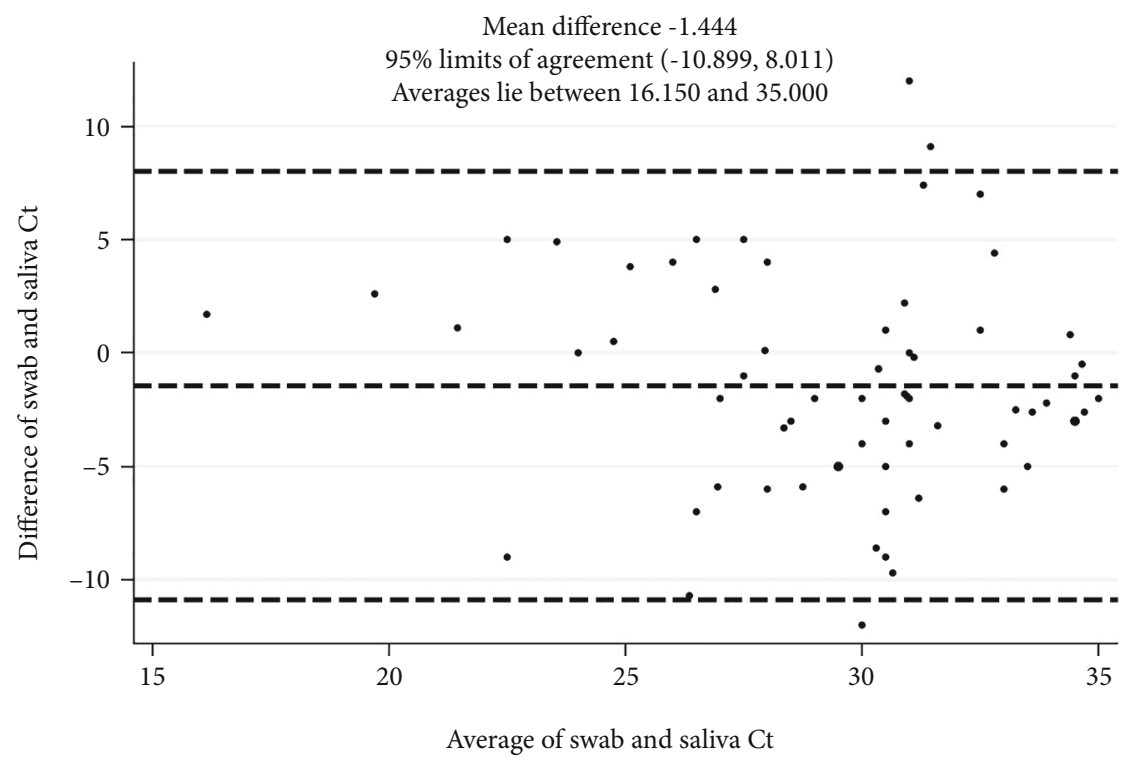

Figure 2: Bland-Altman (B\&A) plot showing concordance between Ct values available for the 71 paired tests.

TABLE 3: Relationship using Cox regression between Ct values where available in both saliva and NP swabs molecular test ( $n=71$ cases) and COVID-19 severity (composite outcome considered as orotracheal intubation or death).

\begin{tabular}{lcccrr}
\hline & HR & $95 \%$ IC & $p$ value & Harrell's C & $95 \%$ IC \\
\hline Ct values on NP swab tests & 0.81 & $0.73-0.89$ & $<0.001$ & 0.738 & $0.628-0.848$ \\
Ct values on saliva samples & 0.89 & $0.83-0.96$ & 0.004 & 0.659 & $0.544-0.774$ \\
\hline
\end{tabular}

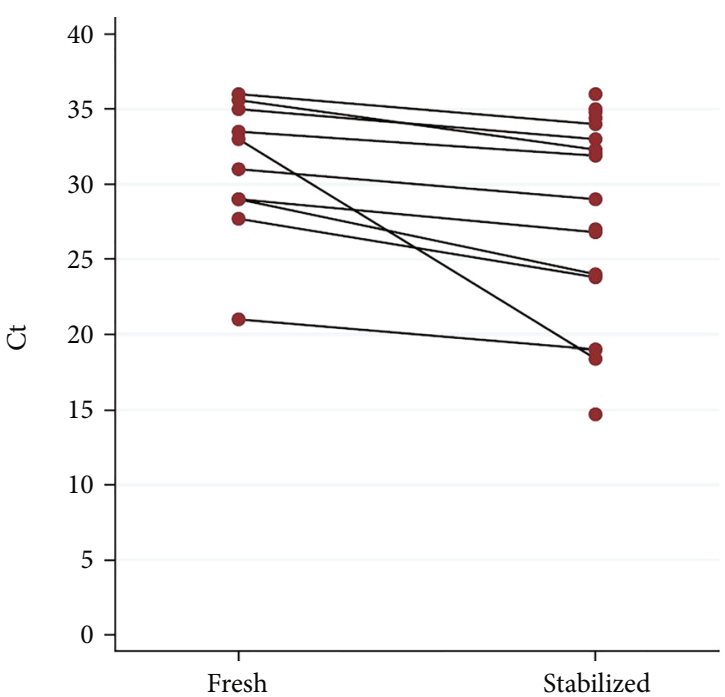

Figure 3: Comparison between Ct values reached from fresh and stabilized saliva samples collected from the same patients.

different dilutions of a positive sample $(\mathrm{Ct}=29)$, with 10 replicates each. CV values reached were always less than the $20 \%$ for all the dilutions tested, which is considered a high degree of accuracy (ISO 3534-1, ISO 5725-1).

The method's precision was evaluated based on intra-assay variation and intermediate precision (interassay variation), expressed as standard deviation $(\sigma)$ or Relative Variation
Coefficient (RDS) and derived from differences between measurement $(\mathrm{Ct})$ means. Furthermore, RT-PCR analysis was repeated on 3 different days to determine the interday precision. Results obtained always revealed RDS values below the $5 \%$, demonstrating a high level of precision (UNI ISO 5725-2: 2020; 3534-1: 2020).

Linearity was determined by serial dilutions of standard samples with known viral copies (synthetic viral sequence) in order to define reportable range. The curve plot of 8 serial dilutions (from $1.00+E^{6}$ to 2 copies) displayed a good linear range estimated as a goodness-of-fit $\left(R^{2}\right)$ equal to 0.996 , indicating a good linear relationship between expected and observed results.

The LoD of the assay for the E-gene at 95\% confidence was 5 copies per reaction, which corresponded to a $\mathrm{Ct}$ value of $36.15 \pm 0.51$.

\section{Discussion and Conclusions}

RT-PCR performed on NP swabs is still considered the gold standard for the diagnosis of SARS-CoV-2 infection $[1,2,8$, 11]. However, the collection of NP swabs is invasive, ideally requires experience and clear instructions and has a risk of viral transmission to the sample collector [11, 14, 23]. There is a need to prioritize the test for people with symptoms of COVID-19 and contacts of those with confirmed SARSCoV-2 infection but also to people at increased risk for exposure (for example, health care workers) [2, 6]; furthermore, there are economic needs as well as the reopening of 
schools maybe they will put a strain on testing systems [6]. The presence of infectious SARS-CoV-2 virus in saliva in asymptomatic or presymptomatic individuals has been demonstrated $[9,24,34,35]$, and this supports the applicability of saliva as sample material for COVID-19 testing [15, 22]. SARS-CoV-2 can be detected in the saliva of COVID-19 patients $[17,24,28]$; studies confirm that saliva is effective for the identification of the SARS-CoV-2 and show higher concentration of RNA viral copies than nasopharyngeal swabs in the same individuals $[17,22,26,28]$.

Saliva samples can sometimes be difficult to handle with existing RNA extraction methods and equipment, and the heterogeneity of saliva specimen can represent limitations, but there are several advantages $[14,31,32,36]$. Actually, saliva sample collection is noninvasive and can be easily performed by the individual themselves, and this could reduce the risk of transmission to the sample takers [13, 14, 17]. In our study, a high-concentrate saline solution was employed to collect saliva samples. The ionic strength of the solution acts as denaturing agent and destroys all protein and muco-protein's structure, interfering on the interaction between the virus and the host cell. This observation represents an additional key aspect in mitigate the risk of infection during sample collection and allows to bypass the absence of dedicate spaces for the safe conduction of laboratory activity. In this work, the combination of the saliva self-collection procedure with the inhibiting property of Bsawb solution could be considered an advancement to facilitate COVID-19 diagnostics.

Furthermore, saliva collection facilitates sampling of children or disabled, and this can increase the acceptance to routine testing practices performed at repeated intervals among high-risk populations. This modality of sample collection is easy and noninvasive, so it may be performed by nonhealthcare professionals or individuals themselves who are properly instructed $[13,14,16,35]$.

Overall, recent studies suggest that the diagnostic sensitivity of RT-PCR on saliva samples is variable [35], often lower but sometimes higher than that of NP swabs, and sensitivity varies when considering different saliva collection technique $[29,37]$. In fact, this variability is often related to an inappropriate saliva sampling $[13,14,36,38]$. In our study, the collection of saliva in a saline solution able to inactivate proteases allowed to recover false negative cases derived from an incorrect preanalytical sampling by NP swab. Saliva degrades very quickly, and this could result in a higher quantity of negative tests, affecting test sensitivity $[31,38,39]$. The constituents of saliva can significantly affect the quality of viral RNA indeed. RNA is very labile and sensitive to the degradation caused by RNAse and endonuclease activity as well as temperature, which can reduce RNA concentration available for the molecular downstream applications [12,31]. Improving the performance of the current saliva collection procedures had the advantage to obtain high-quality RNA for downstream RTPCR test $[14,36,36]$. Furthermore, the stability of nucleic acids in the saline solution guarantees the quality of salivary RNA also as stored at room temperature for 48 hours.

All these advantages allowed to validate the SARS-CoV-2 molecular test on saliva samples in a cohort of 1161 subjects, composed by both patients and controls.
Our experience demonstrated that the clinical sensitivity and specificity reached by test on saliva were $95.1 \%$ (95\% CI 89-98.4\%) and 97.8\% (95\% CI 96.8-98.6\%), respectively, referring to NP swab as gold standard and that agreement between the two tests was very high (97.6\%, Cohen's Kappa coefficient of $0.862,95 \%$ CI $0.812-0.912$ ).

Our data show also that concordance between tests was observed to be associated with subject's age and that in the discordant was 70 years (IQR 61-76) with a significant difference $(p<0.001)$, but this is because the control group was made up of younger and healthier people individuals who underwent screening tests, while the cases were patients hospitalized due to COVID-19.

Taking into account all data, with this study, we demonstrated that saliva could be interchangeably and equally applicable for the identification of SARS-CoV-2 infection, for both diagnostic and screening intents.

In the study by Silva et al., not yet peer-reviewed and published as a preprint, saliva viral load seems to correlate with a spectrum of disease severity throughout the course of illness, and it appears as a predictor of mortality [8].

In our work, we found that COVID-19 severity (composite outcome considered as admission to ICU or death) was correlated with the $\mathrm{Ct}$ values found in both saliva and NP swabs: higher Ct values were considered a protective, meaning that very low levels of viral RNA were found. This analysis showed slightly better performance for the NP swabs, and this could be attributable to a faster viral load clearance in NP swabs than in saliva specimen, which with the high sensitivity demonstrated in this work, it can show a prolonged positivity for viral RNA.

This study, however, was conducted in a period in which the epidemic in our region was sustained by the wild-type strain: it is possible that with the arrival of variants that compartmentalize more in saliva, the results may differ from those found and show better performance for saliva in predicting clinical outcome $[9,40,41]$.

In conclusion, we demonstrated the diagnostic value of saliva as an alternative matrix for SARS-CoV-2 molecular detection. RT-PCR performed on saliva could be applied to the established methods and may provide an additional back-up for population screening. The collection of saliva in a conserving saline solution by patients themselves makes the test more affordable and widely available, preventing the spread of the infection. The test may also look for viral proteins to screen large numbers of asymptomatic people, and in a future perspective, it can be used also to characterize SAR-CoV-2 genetic variants, which are considered more transmissible for their compartmentalization in saliva, their spread, and high replication rate.

\section{Abbreviations}

COVID-19: Coronavirus disease 19

SARS-CoV-2: Severe acute respiratory syndrome coronavirus 2

NP: $\quad$ Nasopharyngeal swab

RT-PCR: Reverse transcription polymerase chain reaction 


$\begin{array}{ll}\text { PPV: } & \text { Positive predicted value } \\ \text { NPV: } & \text { Negative predicted value } \\ \text { Ct: } & \text { Cycle threshold } \\ \text { ICU: } & \text { Intensive care unit } \\ \text { LoD: } & \text { Limit of detection } \\ \text { RT: } & \text { Room temperature } \\ \text { CI: } & \text { Confidence intervals } \\ \text { SD: } & \text { Standard deviation } \\ \text { IQR: } & \text { Interquartile range } \\ \text { HR: } & \text { Hazard ratio. }\end{array}$

\section{Data Availability}

All relevant data are included in the article and materials are available on request.

\section{Disclosure}

An earlier version of this work was presented as an abstract at the EuroMedLab Conference Year 2021, and it is available at [42]. A preprint has previously been published in [43].

\section{Conflicts of Interest}

The authors declare that they have no competing interests.

\section{Authors' Contributions}

MB optimized and performed the experimental analysis and drafted the manuscript. ES coordinated the patient recruitment, collected clinical samples, created and updated the clinical database, and helped to draft the manuscript. BM participated to the experiment design and helped in method's validation. MDM performed the statistical analysis. DC participated in the analysis and in data interpretation. CDC and JDP collected clinical samples and updated the clinical database. RM and AM helped in clinical specimen processing. EN helped in the experimental set up and reviewed the manuscript. CT coordinated the patient recruitment and supervised the study and drafted the manuscript. MI designed the study and performed the statistical analysis. FC conceived, supervised, and coordinated the study and drafted the manuscript. All authors reviewed and approved the final manuscript. MB and ES contributed equally to this work.

\section{Acknowledgments}

The authors are thankful to Biofarma Srl for providing the collection devices employed in the study. This research was funded by PRIN 2017 (n. 20178S4EK9)-“Innovative statistical methods in biomedical research on biomarkers: from their identification to their use in clinical practice."

\section{References}

[1] CDC, Coronavirus Disease 2019 (COVID-19), Centers for Disease Control and Prevention, USA, 2020.
[2] A. K. Bhattacharya and A. Maiti, "Coronavirus disease-2019: an overview and update," Bengal Physician Journal, vol. 6, pp. 45-47, 2019.

[3] M. Uddin, F. Mustafa, T. A. Rizvi et al., "SARS-CoV-2/ COVID-19: viral genomics, epidemiology, vaccines, and therapeutic interventions," Viruses, vol. 12, no. 5, p. 526, 2020.

[4] Commissioner O of the, "Coronavirus (COVID-19) Update: FDA Issues Emergency Use Authorization to Yale School of Public Health for SalivaDirect," in Which Uses a New Method of Saliva Sample Processing, FDA, 2020.

[5] W. Wang, Y. Xu, R. Gao et al., "Detection of SARS-CoV-2 in different types of clinical specimens," JAMA, vol. 323, no. 18, pp. 1843-1844, 2020.

[6] A. Aleta, D. Martín-Corral, A. Pastore y Piontti et al., "Modelling the impact of testing, contact tracing and household quarantine on second waves of COVID-19," Nature Human Behaviour, vol. 4, no. 9, pp. 964-971, 2020.

[7] Centers for Disease Control and Prevention, "Updated Interim Guidelines for Collecting, Handling, and Testing Clinical Specimens from Persons Under Investigation (PUIs) for Coronavirus Disease 2019 (COVID-19)," 2020.

[8] A. L. Wyllie, J. Fournier, A. Casanovas-Massana et al., "Saliva or nasopharyngeal swab specimens for detection of SARSCoV-2," The New England Journal of Medicine, vol. 383, no. 13, pp. 1283-1286, 2020.

[9] Y. Yang, M. Yang, C. Shen et al., "Evaluating the accuracy of different respiratory specimens in the laboratory diagnosis and monitoring the viral shedding of 2019-nCoV infections," medRxiv, vol. 2, no. 11, 2020.

[10] N. Sethuraman, S. S. Jeremiah, and A. Ryo, "Interpreting diagnostic tests for SARS-CoV-2," JAMA, vol. 323, no. 22, pp. 2249-2251, 2020.

[11] K. E. Hanson, A. M. Caliendo, C. A. Arias et al., "Infectious Diseases Society of America guidelines on the diagnosis of Coronavirus Disease 2019," Clinical Infectious Diseases, 2020.

[12] F. M. Marty, K. Chen, and K. A. Verrill, "How to obtain a nasopharyngeal swab specimen," The New England Journal of Medicine, vol. 382, no. 22, 2020.

[13] N. Matic, A. Stefanovic, V. Leung et al., "Practical challenges to the clinical implementation of saliva for SARS-CoV-2 detection," European Journal of Clinical Microbiology \& Infectious Diseases, vol. 40, no. 2, pp. 447-450, 2021.

[14] E. Pasomsub, S. P. Watcharananan, K. Boonyawat et al., "Saliva sample as a non-invasive specimen for the diagnosis of coronavirus disease 2019: a cross-sectional study," Clinical Microbiology and Infection, vol. 27, no. 2, pp. 285.e1-285.e4, 2021.

[15] A. Senok, H. Alsuwaidi, Y. Atrah et al., "Saliva as an alternative specimen for molecular COVID-19 testing in community settings and population-based Screening," Infection and Drug Resistance, vol. Volume 13, pp. 3393-3399, 2020.

[16] K. Skolimowska, M. Rayment, R. Jones, P. Madona, L. S. P. Moore, and P. Randell, "Non-invasive saliva specimens for the diagnosis of COVID-19: caution in mild outpatient cohorts with low prevalence," Clinical Microbiology and Infection, vol. 26, no. 12, pp. 1711-1713, 2020.

[17] K. K. To, L. Lu, C. C. Yip et al., "Additional molecular testing of saliva specimens improves the detection of respiratory viruses," Emerging Microbes \& Infections, vol. 6, no. 1, pp. 1-7, 2017.

[18] Y. Tsujimoto, J. Terada, M. Kimura et al., "Diagnostic accuracy of nasopharyngeal swab, nasal swab and saliva swab samples 
for the detection of SARS-CoV-2 using RT-PCR," Infectious Diseases, vol. 53, no. 8, pp. 581-589, 2021.

[19] L. M. Czumbel, S. Kiss, N. Farkas et al., "Saliva as a candidate for COVID-19 diagnostic testing: a meta-analysis," Frontiers in medicine, vol. 7, p. 465, 2020.

[20] C. Dawes, "Considerations in the development of diagnostic tests on saliva," Annals of the New York Academy of Sciences, vol. 694, pp. 265-269, 1993.

[21] L. Azzi, G. Carcano, F. Gianfagna et al., "Saliva is a reliable tool to detect SARS-CoV-2," Journal of Infection, vol. 81, no. 1, pp. e45-e50, 2020.

[22] H. Altawalah, F. AlHuraish, W. A. Alkandari, and S. Ezzikouri, "Saliva specimens for detection of severe acute respiratory syndrome coronavirus 2 in Kuwait: a cross-sectional study," Journal of Clinical Virology, vol. 132, 2020.

[23] J. R. Campbell, A. Uppal, O. Oxlade et al., "Active testing of groups at increased risk of acquiring SARS-CoV-2 in Canada: costs and human resource needs," CMAJ, vol. 192, no. 40, pp. E1146-E1155, 2020.

[24] M. L. Bastos, S. Perlman-Arrow, D. Menzies, and J. R. Campbell, "The sensitivity and costs of testing for SARS-CoV-2 infection with saliva versus nasopharyngeal Swabs," Annals of Internal Medicine, vol. 174, no. 4, pp. 501-510, 2021.

[25] H. Al Suwaidi, A. Senok, R. Varghese et al., "Saliva for molecular detection of SARS-CoV-2 in school-age children," Clinical Microbiology and Infection, vol. 27, no. 9, pp. 1330-1335, 2021.

[26] G. Butler-Laporte, A. Lawandi, I. Schiller et al., "Comparison of saliva and nasopharyngeal swab nucleic acid amplification testing for detection of SARS-CoV-2," JAMA Internal Medicine, vol. 181, no. 3, p. 353, 2021.

[27] X. He, E. H. Y. Lau, P. Wu et al., "Author correction: temporal dynamics in viral shedding and transmissibility of COVID-19," Nature Medicine, vol. 26, no. 9, pp. 1491-1493, 2020.

[28] K. K. W. To, C. C. Y. Yip, C. Y. W. Lai et al., "Saliva as a diagnostic specimen for testing respiratory virus by a point-of- care molecular assay: a diagnostic validity study," Clinical Microbiology and Infection, vol. 25, no. 3, pp. 372-378, 2019.

[29] A. J. Jamal, M. Mozafarihashin, E. Coomes et al., "Sensitivity of nasopharyngeal swabs and saliva for the detection of severe acute respiratory syndrome coronavirus 2," Clinical Infectious Diseases, vol. 72, no. 6, pp. 1064-1066, 2021.

[30] S. Kernéis, C. Elie, J. Fourgeaud et al., "Accuracy of antigen and nucleic acid amplification testing on saliva and naopharyngeal samples for detection of SARS-CoV-2 in ambulatory care," medRxiv, 2021.

[31] D. Esser, G. Alvarez-Llamas, M. P. de Vries, D. Weening, R. J. Vonk, and H. Roelofsen, "Sample stability and protein composition of saliva: implications for its use as a diagnostic fluid," Biomarker Insights, vol. 3, 2008.

[32] E. Kaufman and I. B. Lamster, "TheDiagnosticApplications ofSaliva- A review," Critical Reviews in Oral Biology and Medicine, vol. 13, no. 2, pp. 197-212, 2002.

[33] W.-L. Guo, Q. Jiang, F. Ye et al., "Effect of throat washings on detection of 2019 novel coronavirus," Clinical Infectious Diseases, vol. 71, no. 8, pp. 1980-1981, 2020.

[34] N. Huang, P. Pérez, T. Kato et al., "SARS-CoV-2 infection of the oral cavity and saliva," Nature Medicine, vol. 27, no. 5, pp. 892-903, 2021.

[35] I. Yokota, P. Y. Shane, K. Okada et al., "Mass screening of asymptomatic persons for SARS-CoV-2 using saliva," Clinical Infectious Diseases, vol. 73, 2021.
[36] D. R. E. Ranoa, R. L. Holland, F. G. Alnaji et al., "Saliva-based molecular testing for SARS-CoV-2 that bypasses RNA extraction," BioRxiv, 2020.

[37] T. J. O'Leary, "Relative sensitivity of saliva and upper airway swabs for initial detection of severe acute respiratory syndrome coronavirus 2 (SARS-CoV-2) in ambulatory patients: rapid review," The Journal of Molecular Diagnostics, vol. 23, no. 3, pp. 265-273, 2021.

[38] I. M. Ott, M. S. Strine, A. E. Watkins et al., "Stability of SARSCoV-2 RNA in nonsupplemented saliva," Emerging Infectious Diseases, vol. 27, no. 4, pp. 1146-1150, 2021.

[39] J. L. Chicharro, A. Lucía, M. Pérez, A. F. Vaquero, and R. Ureña, "Saliva composition and exercise," Sports Medicine, vol. 26, no. 1, pp. 17-27, 1998.

[40] C. K. Wibmer, F. Ayres, T. Hermanus et al., "SARS-CoV-2 501Y.V2 escapes neutralization by South African COVID-19 donor plasma," BioRxiv, 2021.

[41] J. G. Yoon, J. Yoon, J. Y. Song et al., "Clinical significance of a high SARS-CoV-2 viral load in the saliva," Journal of Korean Medical Science, vol. 35, no. 20, 2020.

[42] https://www.degruyter.com/document/doi/10.1515/cclm2021-5028/pdf.

[43] M. Bulfoni, E. Sozio, B. Marcon et al., "Validation of a salivabased test for the molecular diagnosis of SARS-CoV-2 infection," MedRxiv, 2021. 